\title{
RANDOM MINIMALITY AND CONTINUITY OF INVARIANT GRAPHS IN RANDOM DYNAMICAL SYSTEMS
}

\author{
T. JÄGER AND G. KELLER
}

\begin{abstract}
We study dynamical systems forced by a combination of random and deterministic noise and provide criteria, in terms of Lyapunov exponents, for the existence of random attractors with continuous structure in the fibres. For this purpose, we provide suitable random versions of the semiuniform ergodic theorem and also introduce and discuss some basic concepts of random topological dynamics.
\end{abstract}

\section{INTRODUCTION}

Our aim is to introduce a mathematical framework for the investigation of dynamical systems forced by a combination of both deterministic and random external factors and to discuss conceptual issues that arise in this context. In general, dynamical systems under the influence of external forcing are modeled, in discrete time, as skew products

$$
T: \Omega \times M \rightarrow \Omega \times M, \quad T(\omega, x)=\left(\theta \omega, T_{\omega}(x)\right),
$$

where the dynamics of the forcing process are described by the base transforma$\operatorname{tion} \theta: \Omega \rightarrow \Omega$. Note that for simplicity we write $\theta \omega$ instead of $\theta(\omega)$. Typically, depending on the nature of the forcing, $\theta$ is assumed to be either a continuous map of a compact metric space $\Omega$ (deterministic forcing) or a measure-preserving transformation of a probability space $(\Omega, \mathcal{F}, \mathbb{P})$ (random forcing). In a similar way, modeling in continuous time leads to skew product flows, which again give rise to skew product systems of the form (1.1) via their time-one maps.

An invariant graph of $T$ is the graph of a measurable function $\varphi: \Omega \rightarrow M$ which satisfies

$$
T_{\omega}(\varphi(\omega))=\varphi(\theta \omega)
$$

for all (or $\mathbb{P}$-almost all) $\omega \in \Omega$. More generally, invariant graphs may also be multivalued, that is, consist of a constant number $n \geqslant 2$ of points in each fibre. In the study of forced or non-autonomous dynamical systems of the above form, invariant graphs play a central role since they are the natural substitutes of fixed points of autonomous systems. Lyapunov exponents yield additional information about the stability and attractivity of invariant graphs. When $M$ is a smooth manifold and the fibre maps $T_{\omega}$ are all differentiable, the maximal Lyapunov exponent of $\varphi$ with respect to a $\theta$-invariant probablity measure $\mathbb{P}$ is defined as

$$
\lambda_{m}(\varphi)=\lim _{n \rightarrow \infty} \frac{1}{n} \int_{\Omega} \log \left\|D T_{\omega}^{n}(\varphi(\omega))\right\| d \mathbb{P}(\omega) .
$$

Received by the editors July 9, 2013 and, in revised form, September 4, 2014.

2010 Mathematics Subject Classification. Primary 37A30, 37H15, 34D45. 
Here $T_{\omega}^{n}=T_{\theta^{n-1} \omega} \circ \cdots \circ T_{\omega}$ and $D T_{\omega}^{n}(x)$ denotes the derivative of $T_{\omega}^{n}$ in $x$. Note that the limit in (1.3) exists by Kingman's Ergodic Theorem. For the interpretation of the Lyapunov exponents in deterministically forced systems, the continuity of the invariant graph is crucial. In particular, continuous invariant graphs with only negative Lypunov exponents are known to be uniformly attracting and hence stable under perturbation, which is not true in the non-continuous case. A context in which the attractivity of invariant graphs plays a central role is generalised synchronisation, a phenomenon that has been widely studied in theoretical physics [14] and, more recently, also in mathematics [5. The transition from continuous to non-continuous invariant graphs is of interest in the context of non-autonomous bifurcation theory and has equally been studied intensively in different forms, such as fractalisation and torus collision [6].

A criterion ensuring the existence of continuous invariant graphs has been provided by Sturman and Stark in [7]. In order to state it, we define the maximal Lyapunov exponent of a $T$-invariant probability measure $\mu$ as

$$
\lambda(\mu, T)=\lim _{n \rightarrow \infty} \frac{1}{n} \int_{\Omega} \log \left\|D T_{\omega}^{n}(x)\right\| d \mu(\omega, x) .
$$

Note that (1.3) is a special case of (1.4), with $\mu$ given by $\mu(A)=m(\{\omega \in \Omega \mid$ $(\omega, \varphi(\omega)) \in A\}$.

Theorem 1.1 ([7], Theorem 1.14). Suppose $\theta$ is a minimal homeomorphism of a compact metric space $\Omega$ and $f$ is a skew product map of the form (1.1) with $M=\mathbb{R}^{d}$ such that the fibre maps $T_{\omega}$ are differentiable and their derivative depends continuously on $(\omega, x)$. Further, assume that $K$ is a compact $T$-invariant set and $\lambda(\mu, T)<0$ for all $T$-invariant probability measures $\mu$ supported on $K$. Then $K$ is a finite union of continuous invariant graphs (possibly multivalued).

In fact, the above statement is a slight generalisation of the one in 7 and taken from [8, since Sturman and Stark restricted to skew products over irrational translations on a $d$-dimensional torus. In this case, further conclusions can be drawn concerning the regularity of the invariant graphs, which turn out to be as smooth as the system itself; see [9].

In the context of random forcing, one can a priori not speak about the continuity of invariant graphs, due to the lack of a topological structure on the driving space $\Omega$. However, a first question one may ask is whether under similar assumptions a random compact set is just a finite union of invariant graphs, and thus consists of a finite number of points on each fibre. In order to state the respective analogue to Theorem 1.1, we need to introduce some terminology concerning random dynamical systems.

A random map with base $(\Omega, \mathcal{F}, \mathbb{P}, \theta)$, in the sense of Arnold [10, is a skew product of the form (1.1) where $(\Omega, \mathcal{F}, \mathbb{P})$ is a probability space, $\theta: \Omega \rightarrow \Omega$ is a bi-measurable and ergodic measure-preserving bijection and $M$ is a measurable space 1 When $M$ is metric, we always assume that $\mathcal{F}$ is the Borel $\sigma$-algebra on $M$ or its completion. Further, we require the fibre maps $T_{\omega}: M \rightarrow M$ to be continuous and, for each $x \in M$, the map $\omega \mapsto T(\omega, x)$ to be $\mathcal{F}$-measurable. If $M$ is a smooth manifold and all fibre maps $T_{\omega}$ are $\mathcal{C}^{r}$, we call $T$ a random $\mathcal{C}^{r}$-map. If the fibre maps are all homeomorphisms, we call $T$ a random homeomorphism. The set of all $T$-invariant probability measures on $\Omega \times M$ is denoted by $\mathcal{M}(T)$, and

\footnotetext{
${ }^{1}$ The base $(\Omega, \mathcal{F}, \mathbb{P}, \theta)$ is called a measure-preserving dynamical system.
} 
the set of $\mu \in \mathcal{M}(T)$ which project to $\mathbb{P}$ by $\mathcal{M}_{\mathbb{P}}(T)$. Given $K \subseteq \Omega \times M$, we let $K(\omega)=\{x \in M \mid(\omega, x) \in K\}$. We say $K$ is a random compact (closed) set if

(i) $K(\omega)=\{x \in M \mid(\omega, x) \in K\}$ is compact (closed) for all $\omega \in \Omega$;

(ii) the functions $\omega \mapsto d(x, K(\omega))$ are measurable for all $x \in M$.

We now have

Theorem 1.2. Suppose $T: \Omega \times \mathbb{R}^{d} \rightarrow \Omega \times \mathbb{R}^{d}$ is a random $\mathcal{C}^{1}$-map with base $(\Omega, \mathcal{F}, \mathbb{P}, \theta)$, the family $\left(x \mapsto \log \left\|D T_{\omega}^{k}(x)\right\|\right)_{\omega \in \Omega}$ is equicontinuous for all $k \in \mathbb{N}$ and $K \subseteq \Omega \times \mathbb{R}^{d}$ is a random compact set such that $\lambda(\mu, T)<0$ for all $\mu \in \mathcal{M}_{\mathbb{P}}^{K}(T)$. Then there exists an integer $n$ such that $\# K(\omega)=n$ for $\mathbb{P}$-a.e. $\omega \in \Omega$.

This theorem is a special case of Corollary 3.2 in Section 3 below.

Now, in the situation where the forcing has a deterministic component it does make sense to ask for the continuous dependence of invariant graphs on the deterministic variables, even if the graph as a whole is random. In order to make this more precise, we consider systems forced by a combination of random and deterministic noise and model them as double skew products of the form

$$
T: \Omega \times \Xi \times \mathbb{R}^{d} \rightarrow \Omega \times \Xi \times \mathbb{R}^{d}, \quad T(\omega, \xi, y)=\left(\theta \omega, g_{\omega}(\xi), h_{\omega, \xi}(y)\right),
$$

where $(\Omega, \mathcal{F}, \mathbb{P}, \theta)$ is a measure-preserving dynamical system and $\Xi$ is a compact metric space. The second component $\Xi$ of the product space corresponds to the deterministic part of the forcing, which we allow to depend on the random noise as well. Note that fibre maps of $T$ acting on $M=\Xi \times \mathbb{R}^{d}$ are given by $T_{\omega}(\xi, y)=$ $\left(g_{\omega}(\xi), h_{\omega, \xi}(y)\right)$.

To our knowledge, such systems have not been studied in the literature before. However, from the point of view of modeling real-world processes, such an overlay of random and deterministic forcing seems very natural. Furthermore, models of this type come up as well in the bifurcation theory of purely random dynamical systems [11], which provided the starting point of the work presented here.

When $(\Omega \times \Xi, \theta \ltimes g)$ is considered as the basis of (1.5), then an invariant graph is a measurable function $\varphi: \Omega \times \Xi \rightarrow \mathbb{R}^{d}$ which satisfies

$$
h_{\omega, \xi}(\varphi(\omega, \xi))=\varphi\left(\theta \omega, g_{\omega}(\xi)\right) .
$$

Again $\varphi$ cannot be continuous in $\omega$, but $\xi \mapsto \varphi(\omega, \xi)$ may be continuous for $\mathbb{P}$-a.e. $\omega \in \Omega$, in which case we call $\varphi$ a random continuous invariant graph. In order to obtain a random analogue to Theorem 1.1, we have to assume the random map

$$
\theta \ltimes g: \Omega \times \Xi \rightarrow \Omega \times \Xi, \quad(\omega, \xi) \mapsto\left(\theta \omega, g_{\omega}(\xi)\right)
$$

to be a random minimal homeomorphism, which we define as follows.

Definition 1.3. The random homeomorphism $\theta \ltimes g$ is minimal if each $(\theta \ltimes g)$ forward invariant random closed set $K$ obeys the following dichotomy:

either : $K(\omega)=\Xi$ for $\mathbb{P}$-a.e. $\omega$,

or $\quad: K(\omega)=\emptyset$ for $\mathbb{P}$-a.e. $\omega$.

Finally, by $\mathcal{K}\left(\mathbb{R}^{d}\right)$ we denote the set of all compact subsets of $\mathbb{R}^{d}$ and equip it with the Hausdorff distance to make it a metric space. Using these notions, we obtain the following theorem. 
Theorem 1.4. Let $T$ be a random map of the form (1.5) and let $K$ be a $T$-invariant random compact set. Suppose that the family $\left((\xi, y) \mapsto \log \left\|D h_{\omega, \xi}^{k}(y)\right\|\right)_{\omega \in \Omega}$ is equicontinuous for all $k \in \mathbb{N}$. Then, if $\lambda(\mu, T)<0$ for all measures $\mu \in \mathcal{M}_{\mathbb{P}}^{K}(T)$, and if $\theta \ltimes g$ is a random minimal homeomorphism on $\Omega \times \Xi$, there exists a (nonrandom) integer $n>0$ such that, for $\mathbb{P}$-a.e. $\omega \in \Omega$,

(i) $\# K(\omega, \xi)=n$ for all $\xi \in \Xi$,

(ii) the map $\xi \mapsto K(\omega, \xi)$ from $\Xi$ to $\mathcal{K}\left(\mathbb{R}^{d}\right)$ is continuous.

Note that (ii) implies that $K$ can be represented as a finite union of random continuous invariant graphs. We restate and prove this statement, in slightly more general form, as Theorem 3.1 in Section 3. As mentioned in Remark 3.7 a slightly weaker result still holds when $g$ is only random transitive, a notion which is also introduced in Section 3. If $K$ is connected in each fibre, then the minimality assumption on $g$ can even be dropped completely (Theorem 3.3).

A crucial ingredient in the proofs of Theorems 1.2 and 1.4 is a random version of the semiuniform ergodic theorem [7,12. Such a result was already proved by Cao 13 , but since we need a non-trivial modification of his statement (our Theorem 2.8) we provide this in Section 2 with an independent proof. From a conceptual point of view, an interesting aspect of these studies is the fact that notions of random topological dynamics, like random minimality defined above and random transitivity introduced in Section 4, come up naturally in our context. Despite the importance of these concepts in autonomous dynamics, it seems that random analogues have not been considered before. Some basic facts are collected in Section 4, although these can merely serve as starting points for the development of a more comprehensive theory of random topological dynamics.

\section{THE RANDOM SEMIUNIFORM ERGODIC THEOREM}

Recall that a sequence of measurable functions $\Phi_{n}: M \rightarrow \mathbb{R}$ is subadditive with respect to a measurable transformation $T: M \rightarrow M$ if

$$
\Phi_{n+m} \leqslant \Phi_{n} \circ T^{m}+\Phi_{m} \text { for all } n, m \in \mathbb{N} .
$$

Let $\mathcal{M}(T)$ denote the set of $T$-invariant Borel probability measures on $M$. Given $\mu \in \mathcal{M}(T)$, (2.1) implies $\mu\left(\Phi_{n+m}\right) \leqslant \mu\left(\Phi_{n}\right)+\mu\left(\Phi_{m}\right)$, such that by subadditivity the limit

$$
\bar{\Phi}_{\mu}:=\lim _{n \rightarrow \infty} \frac{1}{n} \mu\left(\Phi_{n}\right)=\inf _{n \in \mathbb{N}} \frac{1}{n} \mu\left(\Phi_{n}\right)
$$

exists. Kingman's Ergodic Theorem also ensures the $\mu$-a.s. existence of the pointwise limit

$$
\bar{\Phi}(x)=\lim _{n \rightarrow \infty} \frac{1}{n} \Phi_{n}(x),
$$

provided $\Phi_{1}$ is integrable. Further $\mu(\bar{\Phi})=\bar{\Phi}_{\mu}$, and if $\mu$ is ergodic we also have $\bar{\Phi}(x)=\bar{\Phi}_{\mu}$ for $\mu$-a.e. $x \in M$. When $M$ is a compact metric space, $\Phi$ is continuous and $T$ is a continuous and uniquely ergodi 2 map, then this statement can be strengthened by replacing pointwise with semiuniform convergence on $M$ in (2.2). In fact, for many applications, instead of unique ergodicity it suffices to have an upper bound for the limits $\bar{\Phi}_{\mu}$ with respect to all $T$-invariant probability measures

\footnotetext{
${ }^{2}$ That is, there exists a unique $T$-invariant Borel probability measure on $M$.
} 
$\mu$. In particular, this applies when uniform contraction estimates are derived from negative Lyapunov exponents. We have

Theorem 2.1 (Semiuniform Ergodic Theorem, 7, 12]). Let $M$ be a compact metric space, $T: M \rightarrow M$ a continuous transformation and $\left(\Phi_{n}\right)_{n \in \mathbb{N}}$ a subadditive sequence of continuous functions. Suppose that $\lambda \in \mathbb{R}$ satisfies $\bar{\Phi}_{\mu}<\lambda$ for all $\mu \in \mathcal{M}(T)$. Then there exist $n_{0} \in \mathbb{N}$ and $\delta>0$ such that

$$
\frac{1}{n} \Phi_{n}(x) \leqslant \lambda-\delta \quad \text { for all } n \geqslant n_{0}, x \in M
$$

Our aim is to provide a random analogue to this result, which is needed in the proof of Theorem 1.4 in Section 3 . As before, we denote the set of all $T$-invariant probability measures on $\Omega \times M$ by $\mathcal{M}(T)$, and the set of $\mu \in \mathcal{M}(T)$ which project to $\mathbb{P}$ by $\mathcal{M}_{\mathbb{P}}(T)$. We call $\Phi: \Omega \times M \rightarrow \mathbb{R}$ a random continuous function if

(i) the functions $x \mapsto \Phi(\omega, x)$ are continuous for all $\omega \in \Omega$;

(ii) the functions $\omega \mapsto \Phi(\omega, x)$ are measurable for all $x \in M$.

$U \subseteq \Omega \times M$ is a random open set if $U^{c}$ is random closed. A random set $K$ (open or closed) is called forward $T$-invariant if $T_{\omega}(K(\omega)) \subseteq K(\theta \omega)$ for $\mathbb{P}$-almost every $\omega \in \Omega$. $K$ is called $T$-invariant if the inclusion can be replaced by equality. Note that in contrast to random $T$-invariance, the notions of random continuous functions and random open, closed or compact sets do not depend on the measure $\mathbb{P}$. For any forward $T$-invariant random compact set $K$, we denote the set of $\mu \in \mathcal{M}_{\mathbb{P}}(T)$ which are supported on $K$ by $\mathcal{M}_{\mathbb{P}}^{K}(T)$. Given a random continuous function $\Phi$ : $\Omega \times M \rightarrow \mathbb{R}$ and a random compact set $K$, we define

$$
\Phi^{K}(\omega):=\max \{\Phi(\omega, x) \mid x \in K(\omega)\} .
$$

A random variable $C: \Omega \rightarrow \mathbb{R}$ is called adjusted to $\theta$ if $\lim _{|n| \rightarrow \infty} \frac{1}{|n|} C\left(\theta^{n} \omega\right)=0$ P-a.s.

Theorem 2.2 (Random Semiuniform Ergodic Theorem). Let $T: \Omega \times M \rightarrow \Omega \times$ $M$ be a random map with ergodic base $(\Omega, \mathcal{F}, \mathbb{P}, \theta)$. Suppose that $K$ is a forward $T$-invariant random compact set, and that $\left(\Phi_{n}\right)_{n \in \mathbb{N}}$ is a subadditive sequence of random continuous functions with $\left|\Phi_{n}\right|^{K} \in L^{1}(\mathbb{P})$ for all $n \in \mathbb{N}$. Further, assume that $\lambda \in \mathbb{R}$ satisfies $\bar{\Phi}_{\mu}<\lambda$ for all $\mu \in \mathcal{M}_{\mathbb{P}}^{K}(T)$.

Then there exist $\lambda^{\prime}<\lambda$ and an adjusted random variable $C: \Omega \rightarrow \mathbb{R}$ such that

$$
\Phi_{n}(\omega, x) \leqslant C(\omega)+n \lambda^{\prime} \quad \text { for all } n \in \mathbb{N}, \mathbb{P} \text {-a.e. } \omega \in \Omega \text { and all } x \in K(\omega) .
$$

In particular, for $\delta \in\left(0, \lambda-\lambda^{\prime}\right)$ and $\mathbb{P}$-a.e. $\omega \in \Omega$ there exists $n(\omega) \in \mathbb{N}$ such that

$$
\frac{1}{n} \Phi_{n}(\omega, x) \leqslant \lambda-\delta \quad \text { for all } n \geqslant n(\omega) \text { and } x \in K(\omega) .
$$

Remark 2.3. (a) This theorem generalises [12, Theorem 1] and [7, Theorem 1.9] from the deterministic to the random setting. It is slighty more general than the main result in [13, mostly because of our estimate (2.4), but also because our Lemma 2.4 allows us to avoid the assumption that the probability space $(\Omega, \mathcal{F}, \mathbb{P})$ is complete. As estimate (2.4) and its more subtle variant given in Theorem 2.8 below are crucial for various applications, we give a streamlined full proof of both results.

It should be mentioned that [7] also contains a random version of these results (Theorem 1.19), but only in the sense that the statement is made with respect to a fixed reference measure on the base, while the space $\Omega$ is still assumed to 
be compact and both $T$ and $\Phi_{n}$ are required to be continuous. This theorem is therefore not applicable to general randomly forced systems, and the proof avoids the difficulties coming from the lack of a topological structure on $\Omega$ that we have to deal with here.

(b) Note that $C$ is adjusted in the above sense if and only if $e^{C}$ is tempered [10, Definition 4.1.1]. Hence, by [10, Proposition 4.3.3], given $\epsilon>0$ there is a random variable $D_{\epsilon}$ such that

$$
-\epsilon|n|+D_{\epsilon}(\omega) \leqslant D_{\epsilon}\left(\theta^{n} \omega\right) \leqslant \epsilon|n|+D_{\epsilon}(\omega) \text { for all } n \in \mathbb{Z}
$$

and $C \leqslant D_{\epsilon}$, so that

$$
\Phi_{n}(\omega, x) \leqslant D_{\epsilon}(\omega)+n \lambda^{\prime} \quad \text { for } \mathbb{P} \text {-a.e. } \omega \in \Omega \text { and all } x \in K(\omega) .
$$

(c) Theorem 2.2 can easily be extended to subadditive sequences of random continuous functions $\Phi_{n}: \Omega \times M \rightarrow \mathbb{R} \cup\{-\infty\}$; see Remark 2.9. This is particularly important in the light of applications to Lyapunov exponents involving noninvertible linear cocycles or differential matrices.

In order to prove Theorem 2.2, we start by providing some more basic facts on random dynamical systems. The following lemma is easily derived from results in [14], but we include the proof for the convenience of the reader. Note that unlike the related statements [14, Lemma III.39] and [15, Theorem 8.2.11], it does not require completeness of the probability space $(\Omega, \mathcal{F}, \mathbb{P})$.

Lemma 2.4. $\Phi^{K}: \Omega \rightarrow \mathbb{R}$ is measurable, and there is a measurable map $h: \Omega \rightarrow M$ such that $h(\omega) \in K(\omega)$ and $\Phi^{K}(\omega)=\Phi(\omega, h(\omega))$ for all $\omega \in \Omega$.

Proof. For the random compact set $K$ there is a sequence $\left(a_{k}\right)_{k \in \mathbb{N}}$ of measurable maps $a_{k}: \Omega \rightarrow M$ such that $K(\omega)=\operatorname{closure}\left\{a_{k}(\omega) \mid k \in \mathbb{N}\right\}$ for all $\omega \in \Omega$ [14, Theorem III.30]; see also [10, Proposition 1.6.3]. Therefore, observing the random continuity of $\Phi$,

$$
\Phi^{K}(\omega)=\max \{\Phi(\omega, x) \mid x \in K(\omega)\}=\sup \left\{\Phi\left(\omega, a_{k}(\omega)\right) \mid k \in \mathbb{N}\right\}
$$

is measurable. For $\ell \in \mathbb{N}$ let $k_{\ell}(\omega)=\min \left\{k \in \mathbb{N} \mid \Phi\left(\omega, a_{k}(\omega)\right)>\Phi^{K}(\omega)-\ell^{-1}\right\}$. The $k_{\ell}: \Omega \rightarrow \mathbb{N}$ are measurable. Therefore,

$$
H(\omega)=\bigcap_{j \in \mathbb{N}} \overline{\bigcup_{\ell \geqslant j}\left\{a_{k_{\ell}(\omega)}(\omega)\right\}} \subseteq\left\{x \in K(\omega) \mid \Phi(\omega, x)=\Phi^{K}(\omega)\right\}
$$

is a random compact set [14, Proposition III.4], and there is a measurable selection $h: \Omega \rightarrow M$ such that $h(\omega) \in H(\omega) \subseteq K(\omega)$ for all $\omega \in \Omega$ [14, Theorem III.9].

The proof of the next lemma is straightforward.

Lemma 2.5. If the sequence $\left(\Phi_{n}\right)_{n \in \mathbb{N}}$ of random continuous functions is subadditive and $K$ is a forward invariant random compact set, then the sequence $\left(\Phi_{n}^{K}\right)_{n \in \mathbb{N}}$ is subadditive.

From now on, we use the hypothesis of Theorem 2.2 as standing assumptions for the remainder of this section. Subadditivity of $\left(\Phi_{n}^{K}\right)_{n \in \mathbb{N}}$ allows us to define

$$
\bar{\Phi}^{K}=\inf _{n \in \mathbb{N}} \frac{1}{n} \mathbb{P}\left(\Phi_{n}^{K}\right)=\lim _{n \rightarrow \infty} \frac{1}{n} \mathbb{P}\left(\Phi_{n}^{K}\right) .
$$

Obviously $\bar{\Phi}_{\mu} \leqslant \bar{\Phi}^{K}$ for each $\mu \in \mathcal{M}_{\mathbb{P}}^{K}(f)$. The proofs of the following two results are inspired by the proofs of Lemmas 3 and 4 in [16]. Note that any measure 
$\mu \in \mathcal{M}_{\mathbb{P}}(T)$ can be disintegrated into a family of probability measures $\left(\mu_{\omega}\right)_{\omega \in \Omega}$ on the fibres, in the sense that $\int_{\Omega \times M} \Phi d \mu=\int_{\Omega} \int_{M} \Phi(\omega, x) d \mu_{\omega}(x) d \mathbb{P}(\omega)$ for all measurable functions $\Phi: \Omega \times M \rightarrow \mathbb{R}$ [10, Proposition 1.4.3].

Lemma 2.6. We have $\bar{\Phi}^{K}=\sup \left\{\bar{\Phi}_{\mu} \mid \mu \in \mathcal{M}_{\mathbb{P}}^{K}(T)\right\}$, and the supremum is attained by some $\mu^{*} \in \mathcal{M}_{\mathbb{P}}^{K}(T)$.

Proof. Let $h_{n}$ be measurable selections such that $\Phi_{n}^{K}(\omega)=\Phi_{n}\left(\omega, h_{n}(\omega)\right)$; see Lemma 2.4 Define measures $\mu_{n} \in \mathcal{M}_{\mathbb{P}}^{K}(T)$ via their fibre measures

$$
\mu_{n, \omega}(\theta)=\frac{1}{n} \sum_{i=0}^{n-1} \delta_{T_{\theta^{-i} \omega}^{i}}\left(h_{n}\left(\theta^{-i} \omega\right)\right),
$$

where $\delta_{x}$ denotes the Dirac measure in a point $x \in M$. As $h_{n}(\omega) \in K(\omega)$ for each $\omega \in \Omega$, all measures $\mu_{n}$ are supported by the forward invariant random compact set $K$. Hence, by the random Krylov-Bogulyubov Theorem ([17] or [10, Theorem 1.6.13]), there is a subsequence $\left(\mu_{n_{l}}\right)_{l \in \mathbb{N}}$ converging (random-)weakly to some $\mu^{*} \in$ $\mathcal{M}_{\mathbb{P}}^{K}(f)$.

Now fix $k \in \mathbb{N}$. Then, for some $t \in\{0, \ldots, k-1\}$ the sequence $\left(n_{l}\right)_{l \in \mathbb{N}}$ contains a subsequence of the form $\left(s_{j} k+t\right)_{j \in \mathbb{N}}$. Note that for any sequence $\left(x_{j}\right)_{j \in \mathbb{N}}$ of measurable functions $x_{j}: \Omega \rightarrow M$ with $x_{j}(\omega) \in K(\omega)$ for all $\omega \in \Omega, j \in \mathbb{N}$, and any sequence of integers $\left(N_{j}\right)_{j \in \mathbb{N}}$ with $N_{j} \nearrow \infty$, the fact that $\left|\Phi_{k}\right|^{K} \in L^{1}(\mathbb{P})$ easily implies

$$
\lim _{j \rightarrow \infty} \frac{1}{N_{j}} \int_{\Omega} \Phi_{k}\left(\omega, x_{j}(\omega)\right) d \mathbb{P}(\omega)=0
$$

Using this observation, we obtain

$$
\begin{aligned}
\mu^{*}\left(\frac{1}{k} \Phi_{k}\right) & =\lim _{j \rightarrow \infty} \frac{1}{k} \int_{\Omega} \Phi_{k} d \mu_{s_{j} k+t} \\
& =\lim _{j \rightarrow \infty} \frac{1}{k\left(s_{j} k+t\right)} \sum_{i=0}^{s_{j} k+t-1} \int_{\Omega} \Phi_{k} \circ T^{i}\left(\theta^{-i} \omega, h_{s_{j} k+t}\left(\theta^{-i} \omega\right)\right) d \mathbb{P}(\omega) \\
& =\lim _{j \rightarrow \infty} \frac{1}{k\left(s_{j} k+t\right)} \sum_{i=0}^{s_{j} k+t-1} \int_{\Omega} \Phi_{k} \circ T^{i}\left(\omega, h_{s_{j} k+t}(\omega)\right) d \mathbb{P}(\omega) \\
& \stackrel{2.6}{=} \lim _{j \rightarrow \infty} \frac{1}{k\left(s_{j} k+t\right)} \sum_{i=0}^{k-1} \sum_{l=0}^{s_{j}-2} \int_{\Omega} \Phi_{k} \circ T^{k l+i}\left(\omega, h_{s_{j} k+t}(\omega)\right) d \mathbb{P}(\omega) \\
& \geqslant \lim _{j \rightarrow \infty} \frac{1}{k\left(s_{j} k+t\right)} \sum_{i=0}^{k-1} \int_{\Omega} \Phi_{\left(s_{j}-1\right) k} \circ T^{i}\left(\omega, h_{s_{j} k+t}(\omega)\right) d \mathbb{P}(\omega) \\
& \lim _{j \rightarrow \infty} \frac{1}{k\left(s_{j} k+t\right)} \sum_{i=0}^{k-1}\left(\int_{\Omega} \Phi_{s_{j} k+t}\left(\omega, h_{s_{j} k+t}(\omega)\right) d \mathbb{P}(\omega)\right. \\
& \left.-\int_{\Omega} \Phi_{i}\left(\omega, h_{s_{j} k+t}(\omega)\right)+\Phi_{k-i+t} \circ T^{\left(s_{j}-1\right) k+i}\left(\omega, h_{s_{j} k+t}(\omega)\right) d \mathbb{P}(\omega)\right) \\
& \lim _{j \rightarrow \infty} \frac{1}{s_{j} k+t} \int_{\Omega} \Phi_{s_{j} k+t}\left(\omega, h_{s_{j} k+t}(\omega)\right) d \mathbb{P}(\omega) \\
& \lim _{j \rightarrow \infty} \frac{1}{s_{j} k+t} \int_{\Omega} \Phi_{s_{j} k+t}^{K}(\omega) d \mathbb{P}(\omega) \quad=\quad \Phi^{K} . \\
&
\end{aligned}
$$


Since this holds for all $k \in \mathbb{N}$, we have $\bar{\Phi}_{\mu^{*}}=\inf _{k \in \mathbb{N}} \mu^{*}\left(\frac{1}{k} \Phi_{k}\right) \geqslant \bar{\Phi}^{K}$.

For the proof of Theorem 2.2, we will further need the following useful criterion for the adjustedness of random variables.

Lemma 2.7. Suppose $C: \Omega \rightarrow \mathbb{R}$ is measurable and $C \circ \theta-C$ has a $\mathbb{P}$-integrable minorant. Then $C$ is adjusted to $\theta$.

Proof. Since $C \circ \theta-C$ has an integrable minorant, [18, Lemma 4.1.13] implies that $C \circ \theta-C \in L^{1}(m)$ and $\int_{\Omega} C \circ \theta-C d m=0$. Hence, we obtain from the Birkhoff Ergodic Theorem that

$$
\begin{aligned}
\lim _{n \rightarrow \infty} \frac{1}{n} C\left(\theta^{n} \omega\right) & =\lim _{n \rightarrow \infty} \frac{1}{n}\left(C\left(\theta^{n} \omega\right)-C(\omega)\right)=\lim _{n \rightarrow \infty} \frac{1}{n} \sum_{i=0}^{n-1} C\left(\theta^{i+1} \omega\right)-C\left(\theta^{i} \omega\right) \\
& =\lim _{n \rightarrow \infty} \frac{1}{n} \sum_{i=0}^{n-1}(C \circ \theta-C) \circ \theta^{i}(\omega)=0
\end{aligned}
$$

for $m$-a.e. $\omega \in \Omega$. Since $C \circ \theta^{-1}-C=-(C \circ \theta-C) \circ \theta^{-1} \in L^{1}(\mathbb{P})$ as well, the limit for $n \rightarrow-\infty$ can be treated in the same way.

Proof of Theorem 2.2. Suppose $\lambda>\bar{\Phi}_{\mu}$ for all $\mu \in \mathcal{M}_{\mathbb{P}}^{K}(T)$. Then $\lambda>\bar{\Phi}^{K}$ by Lemma 2.6, and we can choose $\lambda^{\prime} \in\left(\bar{\Phi}^{K}, \lambda\right)$. We have $\lim _{n \rightarrow \infty} \frac{1}{n} \Phi_{n}^{K}(\omega)=\bar{\Phi}^{K}$ $\mathbb{P}$-a.s. by Kingman's Subadditive Ergodic Theorem. If we let $\Phi_{0}^{K}=0$, then the random variable $C$ defined by

$$
C(\omega)=\sup _{n \geqslant 0}\left(-\lambda^{\prime} n+\Phi_{n}^{K}(\omega)\right)
$$

is non-negative and $\mathbb{P}$-a.s. finite. The fact that $C$ satisfies $(2.4)$ is obvious from its definition. Further, if $C(\omega)=0$, then $C(\theta \omega)-C(\omega) \geqslant 0$. Otherwise, since

$$
\begin{aligned}
-\lambda^{\prime} n+\Phi_{n}^{K}(\omega) & \leqslant\left(-\lambda^{\prime}(n-1)+\Phi_{n-1}^{K}(\theta \omega)\right)+\left(-\lambda^{\prime}+\Phi_{1}^{K}(\omega)\right) \\
& \leqslant C(\theta \omega)-\lambda^{\prime}+\Phi_{1}^{K}(\omega),
\end{aligned}
$$

for all $n \geqslant 1$, we have that

$$
C(\omega) \leqslant C(\theta \omega)-\lambda^{\prime}+\Phi_{1}^{K}(\omega) .
$$

Combining both estimates yields

$$
C(\theta \omega)-C(\omega) \geqslant \min \left\{0, \lambda^{\prime}-\Phi_{1}^{K}(\omega)\right\} .
$$

Hence $C \circ \theta-C$ has an integrable minorant, and thus $C$ is adjusted to $\theta$ by Lemma 2.7.

For the proof of Theorem 1.4 the following variation of Theorem 2.2 will be crucial.

Theorem 2.8. In the situation of Theorem 2.2, there exist $\lambda^{\prime}<\lambda$ and $k_{0} \in \mathbb{N} \backslash\{0\}$ such that for all $k \geqslant k_{0}$ there are an adjusted random variable $\widehat{C}_{k}: \Omega \rightarrow[0, \infty)$ and an ergodic componen $\Omega_{k}$ of $\theta^{k}$ with $\mathbb{P}\left(\Omega_{k}\right) \geqslant 1 / k$ such that

$$
\Phi_{k}(\omega, x) \leqslant \widehat{C}_{k}\left(\theta^{k} \omega\right)-\widehat{C}_{k}(\omega)+k \lambda^{\prime} \quad \text { for } \mathbb{P} \text {-a.e. } \omega \in \Omega_{k} \text { and all } x \in K(\omega) \text {. }
$$

\footnotetext{
${ }^{3}$ An ergodic component of $\theta^{k}$ is a $\theta^{k}$-invariant set $\Omega_{k}$ of positive measure such that $\theta_{\mid \Omega_{k}}^{k}$ is ergodic.
} 
The random variables $\widehat{C}_{k}$ can also be chosen to take values in $(-\infty, 0]$. Furthermore,

a) if $\left(\Phi_{n}\right)_{n \in \mathbb{N}}$ is additive 4 then $k_{0}=1$ so that (2.7) holds for $k=1$ and $\mathbb{P}$-a.e. $\omega \in \Omega$

b) if $\theta$ is totally ergodic 5 then (2.7) holds for $\mathbb{P}$-a.e. $\omega \in \Omega$.

Proof. As $\bar{\Phi}^{K}<\lambda$ by Lemma 2.6, there is $k_{0} \in \mathbb{N}$ such that $\mathbb{E}_{\mathbb{P}}\left[\Phi_{k}^{K}\right]=\int_{\Omega} \Phi_{k}^{K} d \mathbb{P}<$ $k \lambda^{\prime}$ for some $\lambda^{\prime}<\lambda$ and all $k \geqslant k_{0}$. Fix any such $k$. Then

$$
\lim _{n \rightarrow \infty} \frac{1}{n} \sum_{j=1}^{n} \Phi_{k}^{K}\left(\theta^{-j k} \omega\right)=\mathbb{E}_{\mathbb{P}}\left[\Phi_{k}^{K} \mid \mathcal{I}_{k}\right](\omega) \text { for } \mathbb{P} \text {-a.e. } \omega,
$$

where $\mathbb{E}_{\mathbb{P}}\left[\cdot \mid \mathcal{I}_{k}\right]$ denotes the conditional expectation w.r.t. the $\sigma$-algebra $\mathcal{I}_{k} \subseteq \mathcal{F}$ of all $\theta^{k}$-invariant sets. Since $\theta$ is ergodic, all sets of positive measure in $\mathcal{I}_{k}$ have measure at least $1 / k$. As $\int_{\Omega} \mathbb{E}_{\mathbb{P}}\left[\Phi_{k}^{K} \mid \mathcal{I}_{k}\right] d \mathbb{P}=\mathbb{E}_{\mathbb{P}}\left[\Phi_{k}^{K}\right]<k \lambda^{\prime}$, this means that there is an ergodic component of $\theta^{k}$ such that $\lim _{n \rightarrow \infty} \frac{1}{n} \sum_{j=1}^{n} \Phi_{k}^{K}\left(\theta^{-j k} \omega\right)<k \lambda^{\prime}$ for $\mathbb{P}$-a.e. $\omega \in \Omega_{k}$. Hence,

$$
0 \leqslant \widehat{C}_{k}(\omega)=\sup _{n \geqslant 0}\left(-\lambda^{\prime} n k+\sum_{j=1}^{n} \Phi_{k}^{K}\left(\theta^{-j k} \omega\right)\right)<\infty \quad \text { for } \mathbb{P} \text {-a.e. } \omega \in \Omega_{k} .
$$

Let $\omega \in \Omega_{k}$. We prove (2.7):

$$
\begin{aligned}
\widehat{C}_{k}\left(\theta^{k} \omega\right) & =\sup _{n \geqslant 0}\left(-\lambda^{\prime} n k+\sum_{j=0}^{n-1} \Phi_{k}^{K}\left(\theta^{-j k} \omega\right)\right) \\
& \geqslant \sup _{n \geqslant 1}\left(-\lambda^{\prime} n k+\sum_{j=0}^{n-1} \Phi_{k}^{K}\left(\theta^{-j k} \omega\right)\right) \\
& =\sup _{n \geqslant 1}\left(-\lambda^{\prime}(n-1) k+\sum_{j=1}^{n-1} \Phi_{k}^{K}\left(\theta^{-j k} \omega\right)\right)-\lambda^{\prime} k+\Phi_{k}^{K}(\omega) \\
& =\widehat{C}_{k}(\omega)-\lambda^{\prime} k+\Phi_{k}^{K}(\omega) .
\end{aligned}
$$

This also implies that $\widehat{C}_{k} \circ \theta^{k}-\widehat{C}_{k}$ has the integrable minorant $-\lambda^{\prime} k+\Phi_{k}^{K}$ so that, in view of [18, Lemma 4.1.13], $\widehat{C}_{k} \circ \theta^{k}-\widehat{C}_{k} \in L_{\mathbb{P}}^{1}$ and $\int \widehat{C}_{k} \circ \theta^{k}-\widehat{C}_{k} d \mathbb{P}=0$. Thus, Lemma 2.7 implies that $\widehat{C}_{k}$ is adapted to $\theta^{k}$. In order to show that it is also adapted to $\theta$, let $\ell \in\{0, \ldots, k-1\}$. Then Birkhoff's Ergodic Theorem implies that

$$
\begin{aligned}
0 & \leqslant \lim _{n \rightarrow \infty} \frac{1}{n k+\ell} \widehat{C}_{k}\left(\theta^{n k+\ell} \omega\right)=\lim _{n \rightarrow \infty} \frac{1}{n} \sum_{j=0}^{n-1} \frac{1}{k}\left(\widehat{C}_{k} \circ \theta^{k}-\widehat{C}_{k}\right)\left(\theta^{j k}\left(\theta^{\ell} \omega\right)\right) \\
& =\frac{1}{k} \mathbb{E}_{\mathbb{P}}\left[\widehat{C}_{k} \circ \theta^{k}-\widehat{C}_{k} \mid \mathcal{I}_{k}\right]\left(\theta^{\ell} \omega\right) \quad \text { for } \mathbb{P} \text {-a.e. } \omega .
\end{aligned}
$$

As $\int \mathbb{E}_{\mathbb{P}}\left[\widehat{C}_{k} \circ \theta^{k}-\widehat{C}_{k} \mid \mathcal{I}_{k}\right] d \mathbb{P}=\int \widehat{C}_{k} \circ \theta^{k}-\widehat{C}_{k} d \mathbb{P}=0$, it follows that the limit is actually equal to zero for $\mathbb{P}$-a.e. $\omega$. Hence $\widehat{C}_{k}$ is adapted to $\theta$. The case $n \rightarrow-\infty$ is similar.

\footnotetext{
${ }^{4}$ That is, 2.1 holds with equality.

${ }^{5}$ That is, $\theta^{k}$ is ergodic for all $k \in \mathbb{N}$.
} 
Observe that the $\widehat{C}_{k}$ are all non-negative. We finally show how to modify the above construction to obtain non-positive $\widehat{C}_{k}$. To this end let

$$
\widehat{C}_{k}(\omega)=-\sup _{n \geqslant 0}\left(-\lambda^{\prime} n k+\sum_{j=0}^{n-1} \Phi_{k}^{K}\left(\theta^{j k} \omega\right)\right) .
$$

As above one shows that there is an ergodic component $\Omega_{k}$ of $\theta^{k}$ such that $-\infty<$ $\widehat{C}_{k}(\omega) \leqslant 0$ for $\mathbb{P}$-a.e. $\omega \in \Omega_{k}$. We prove (2.7):

$$
\begin{aligned}
\widehat{C}_{k}\left(\theta^{k} \omega\right) & =\inf _{n \geqslant 0}\left(\lambda^{\prime} n k-\sum_{j=1}^{n} \Phi_{k}^{K}\left(\theta^{j k} \omega\right)\right) \\
& =\inf _{n \geqslant 0}\left(\lambda^{\prime}(n+1) k-\sum_{j=0}^{(n+1)-1} \Phi_{k}^{K}\left(\theta^{j k} \omega\right)\right)-\lambda^{\prime} k+\Phi_{k}^{K}(\omega) \\
& \geqslant \widehat{C}_{k}(\omega)-\lambda^{\prime} k+\Phi_{k}^{K}(\omega) .
\end{aligned}
$$

Again, $\widehat{C}_{k} \circ \theta^{k}-\widehat{C}_{k}$ has the integrable minorant $-\lambda^{\prime} k+\Phi_{k}^{K}$, and as before one proves that $\widehat{C}_{k}$ is adjusted to $\theta$.

Remark 2.9. As mentioned, all the above results can also be applied to subadditive sequences $\left(\Phi_{n}\right)_{n \in \mathbb{N}}$ of random continuous functions taking values in $\mathbb{R} \cup\{-\infty\}$ (equipped with the obvious topology). In order to see this, suppose $\bar{\Phi}_{\mu}<\lambda$ for all ergodic invariant measures $\mu \in \mathcal{M}_{\mathbb{P}}^{K}(T)$. Then Kingman's Subadditive Ergodic Theorem, which still applies since it does not assume continuity of the $\Phi_{n}$, implies that for all ergodic $\mu \in \mathcal{M}_{\mathbb{P}}^{K}(T)$ we have

$$
\limsup _{n \rightarrow \infty} \frac{1}{n} \Phi_{n}<\lambda \mu \text {-a.e. }
$$

Let $\lambda^{\prime}<\lambda$ and $\Phi_{n}^{\prime}(\omega, x)=\max \left\{n \lambda^{\prime}, \Phi_{n}(\omega, x)\right\}$. Then $\left(\Phi_{n}^{\prime}\right)_{n \in \mathbb{N}}$ is subadditive and

$$
\limsup _{n \rightarrow \infty} \frac{1}{n} \Phi_{n}^{\prime}<\lambda \mu \text {-a.e. }
$$

and hence $\bar{\Phi}_{\mu}^{\prime}<\lambda$ for all ergodic $\mu \in \mathcal{M}_{\mathbb{P}}^{K}(T)$. Thus, we can apply the above results to the subadditive sequence $\left(\Phi_{n}^{\prime}\right)_{n \in \mathbb{N}}$ of continuous real-valued functions. Since $\Phi_{n}^{\prime} \geqslant \Phi_{n}$, all estimates then carry over to the original sequence.

\section{CONTINUity OF RANDOM INVARIANT GRAPHS}

We now consider random dynamical systems with a double skew product structure

$$
T: \Omega \times M \rightarrow \Omega \times M, \quad T(\omega, \xi, y)=\left(\theta \omega, g_{\omega}(\xi), h_{\omega, \xi}(y)\right),
$$

where $M=\Xi \times \mathbb{R}^{d}$ as above and $\Xi$ is again a compact metric space. We assume that the maps $g_{\omega}$ are homeomorphisms, the maps $(\xi, y) \mapsto h_{\omega, \xi}(y)$ are continuous and differentiable in $y$ and $D h_{\omega, \xi}(y)$ is continuous in $(\xi, y)$ for all $\omega \in \Omega$. As mentioned, the action of the $g_{\omega}$ on the second component $\Xi$ corresponds to the deterministic part of the forcing, such that the combined forcing process is the random homeomorphism

$$
\theta \ltimes g: \Omega \times \Xi \rightarrow \Omega \times \Xi, \quad \theta \ltimes g(\omega, \xi)=\left(\theta \omega, g_{\omega}(\xi)\right) .
$$


In this way, given any $\theta \ltimes g$-invariant probability measure $m$ we can view $T=$ $(\theta \ltimes g) \ltimes h$ as a random map over the base $(\Omega \times \Xi, \mathcal{F} \times \mathcal{X}, m, \theta \ltimes g)$, where $\mathcal{X}$ denotes the Borel $\sigma$-algebra on $\Xi$. An alternative point of view is to write $T=\theta \ltimes(g \ltimes h)$, thus interpreting $T$ as a random map over the base $(\Omega, \mathcal{F}, \mathbb{P}, \theta)$. In this case the fibre maps $T_{\omega}$ have a skew product structure themselves, and $T_{\omega}: M \rightarrow M$ is a continuous transformation of the form $T_{\omega}(\xi, y)=\left(g_{\omega}(\xi), h_{\omega, \xi}(y)\right)$. Given $T$ as in (3.1) and a random $T$-invariant set $K$, we let $K(\omega)=\{(\xi, y) \in M \mid(\omega, \xi, y) \in$ $K\}$ and $K(\omega, \xi)=\left\{y \in \mathbb{R}^{d} \mid(\omega, \xi, y) \in K\right\}$. Then $T_{\omega}(K(\omega))=K(\theta \omega)$ and $h_{\omega, \xi}(K(\omega, \xi))=K(\theta \ltimes g(\omega, \xi))$ (see Lemma 3.5 below).

Similar to above, the Lyapunov exponent of a $T$-invariant measure $\mu$ is defined as

$$
\lambda(\mu, T)=\mu(\bar{\Phi})=\inf _{n \in \mathbb{N}} \frac{1}{n} \mu\left(\Phi_{n}\right),
$$

where now the subadditive sequence $\Phi_{n}$ is given by $\Phi_{n}(\omega, \xi, y)=\log \left\|D_{y} h_{\omega, \xi}^{n}(y)\right\|$. We will prove the following slightly more general version of Theorem 1.4.

Theorem 3.1. Let $T$ be a random map of the form (3.1) and let $K$ be a $T$-invariant random compact set. Suppose that for all $k \in \mathbb{N}$ and all $\epsilon>0$ there is $r>0$ such that

$$
\begin{aligned}
\sup & \left\{\Phi_{k}(\omega, \xi, y) \mid \omega \in \Omega,(\xi, y) \in B_{r}(K(\omega))\right\} \\
& \leqslant \sup \left\{\Phi_{k}(\omega, \xi, y) \mid \omega \in \Omega,(\xi, y) \in K(\omega)\right\}+\epsilon,
\end{aligned}
$$

where $B_{r}(K(\omega))=\left\{(\xi, y) \in \Xi \times \mathbb{R}^{d} \mid d((\xi, y), K(\omega))<r\right\}$ and $d$ is a canonical product metric on $\xi \times \mathbb{R}^{d}$. Then, if $\lambda(\mu, T)<0$ for all measures $\mu \in \mathcal{M}_{\mathbb{P}}^{K}(T)$, and if $\theta \ltimes g$ is a random minimal homeomorphism on $\Omega \times \Xi$, there are a (non-random) integer $n>0$ and a random variable $c(\omega)>0$ such that, for $\mathbb{P}$-a.e. $\omega \in \Omega$,

(i) $\# K(\omega, \xi)=n$ for all $\xi \in \Xi$,

(ii) the map $\xi \mapsto K(\omega, \xi)$ from $\Xi$ to $\mathcal{K}\left(\mathbb{R}^{d}\right)$ is continuous, and

(iii) for all $\xi \in \Xi$, any two different points $y, y^{\prime} \in K(\omega, \xi)$ have distance at least $c(\omega)$.

If $T: \Omega \times \mathbb{R}^{d} \rightarrow \Omega \times \mathbb{R}^{d}$ is a random map with base $(\Omega, \mathcal{F}, \mathbb{P}, \theta)$, then we can add a trivial component $\Xi=\left\{\xi_{0}\right\}$ and let $g_{\omega}\left(\xi_{0}\right)=\xi_{0}$ to apply Theorem 3.1. As $\theta \ltimes g$ is certainly random minimal in this case, this immediately yields

Corollary 3.2. Suppose $T: \Omega \times \mathbb{R}^{d} \rightarrow \Omega \times \mathbb{R}^{d}$ is a random $\mathcal{C}^{1}$-map with base $(\Omega, \mathcal{F}, \mathbb{P}, \theta)$ and $K \subseteq \Omega \times \mathbb{R}^{d}$ is a random compact set such that (3.4) is satisfied and $\lambda(\mu, T)<0$ for all $\mu \in \mathcal{M}_{\mathbb{P}}^{K}(T)$. Then there exists an integer $n$ such that $\# K(\omega)=n$ for $\mathbb{P}$-a.e. $\omega \in \Omega$.

The minimality assumption on $\theta \ltimes g$ in Theorem 3.1 can also be replaced by requiring connectedness of the sets $K(\omega, \xi)$. We say the random compact set $K \subseteq$ $\Omega \times \Xi \times \mathbb{R}^{d}$ has connected fibres if $K(\omega, \xi)$ is connected for $\mathbb{P}$-a.e. $\omega \in \Omega$ and all $\xi \in \Xi$.

Theorem 3.3. Let $T$ be a random map of the form (3.1) and let $K$ be a $T$ invariant random compact set with connected fibres. Suppose that for all $k \in \mathbb{N}$ and all $\epsilon>0$ there is $r>0$ such that (3.4) holds and that $\lambda(\mu, T)<0$ for all measures $\mu \in \mathcal{M}_{\mathbb{P}}^{K}(T)$. Then for $\mathbb{P}$-a.e. $\omega \in \Omega$ the fibre $K(\omega)$ consists of a single continuous graph; that is, there is a random continuous function $\phi: \Omega \times \Theta \rightarrow \mathbb{R}^{d}$ such that $K(\omega)=\{(\xi, \phi(\omega, \xi)) \mid \xi \in \Xi\}$ for $\mathbb{P}$-a.e. $\omega \in \Omega$. 
Both theorems are consequences of the following more technical proposition. We say an open or closed random set $A \subseteq \Omega \times \Xi$ is non-empty if $\{\omega \in \Omega \mid A(\omega) \neq \emptyset\}$ has positive measure. Note that if $A$ is $(\theta \ltimes g)$-invariant and $\theta$ is ergodic, then non-emptiness of $A$ implies that $\{\omega \in \Omega \mid A(\omega)=\emptyset\}$ has measure zero. Similarly, equality of random sets will always be understood as an equality of the fibres for P-a.e. $\omega \in \Omega$.

Proposition 3.4. Let $T$ be a random map of the form (3.1) and let $K$ be a $T$ invariant random compact set. Suppose that for all $k \in \mathbb{N}$ and all $\epsilon>0$ there is $r>0$ such that (3.4) holds. Then, if $\lambda(\mu, T)<0$ for all measures $\mu \in \mathcal{M}_{\mathbb{P}}^{K}(T)$, there are a positive integer $n$, a random variable $c: \Omega \rightarrow(0, \infty)$ and a non-empty open and $(\theta \ltimes g)$-forward invariant random set $A$ such that, for $\mathbb{P}$-a.e. $\omega \in \Omega$,

(i) $\# K(\omega, \xi)=n$ for all $\xi \in A(\omega)$,

(ii) $\sup \{\# K(\omega, \xi) \mid \xi \in \Xi\}<\infty$, and

(iii) for all $\xi \in \Xi$, any two different points $y, y^{\prime} \in K(\omega, \xi)$ have distance at least $c(\omega)$.

Proof of Theorem 3.1. As $A$ is a non-empty forward $(\theta \ltimes g)$-invariant random set, $A^{c}$ is a backwards $(\theta \ltimes g)$-invariant random compact set and $A^{c}(\omega) \neq \Xi$ for $\mathbb{P}$-a.e. $\omega$. As $\theta \ltimes g$ is minimal, $A^{c}(\omega)=\emptyset$ for $\mathbb{P}$-a.e. $\omega$ (see Lemma 4.5 (ii) below), and hence $A(\omega)=\Xi$ for $\mathbb{P}$-a.e. $\omega$. Assertions (i) and (iii) of the proposition together with the compactness of $K(\omega)$ imply the continuity of the map $\xi \mapsto K(\omega, \xi)$ from $\Xi$ to $\mathcal{K}\left(\mathbb{R}^{d}\right)$.

Proof of Theorem 3.3. By assertion (ii) of the proposition, together with the connectedness of the fibres of $K$, there is a subset $\Omega_{0} \subseteq \Omega$ of full measure such that $K(\omega, \xi) \subset \mathbb{R}^{d}$ consists of a single point for all $(\omega, \xi) \in \Omega_{0} \times \Xi$. As $K(\omega) \subset \Xi \times \mathbb{R}^{d}$ is compact, it must be the graph of a continuous map $\phi(\omega,):. \Xi \rightarrow \mathbb{R}^{d}$. As $\{\phi(\omega, \xi)\}=K(\omega, \xi)$ is the only possible selection of $K, \phi$ is measurable [10, Proposition 1.6.3].

Proof of Proposition 3.4. By Theorem 2.8 there exist $\lambda^{\prime}<0, k \in \mathbb{N} \backslash\{0\}$, an ergodic component $\Omega_{k}$ of $\theta^{k}$, and an adjusted random variable $\widehat{C}: \Omega \rightarrow(-\infty, 0]$ such that

$$
\Phi_{k}(\omega, \xi, y) \leqslant \widehat{C}\left(\theta^{k} \omega\right)-\widehat{C}(\omega)+k \lambda^{\prime} \quad \text { for } \mathbb{P} \text {-a.e. } \omega \in \Omega_{k} \text { and all }(\xi, y) \in K(\omega) .
$$

Hence, in view of assumption (3.4), there are $r>0$ and $\eta>0$ such that

$\log \left\|D_{y} h_{\omega, \xi}^{k}(y)\right\| \leqslant \widehat{C}\left(\theta^{k} \omega\right)-\widehat{C}(\omega)-\eta \quad$ for $\mathbb{P}$-a.e. $\omega \in \Omega_{k}$ and all $(\xi, y) \in B_{r}(K(\omega))$.

Now we consider $T$ as $(\theta \ltimes g) \ltimes h$. We defer the proof of the following two lemmas to the end of this section.

Lemma 3.5. $K$ is a random compact set over the base $(\Omega \times \Xi, \mathcal{F} \times \mathcal{B})$, and $h_{\omega, \xi}(K(\omega, \xi))=K((\theta \ltimes g)(\omega, \xi))$ for $\mathbb{P}$-a.e. all $\omega \in \Omega$ and $\xi \in \Xi$.

Next, for $\epsilon \in(0, r]$, define $N_{\epsilon}(\omega, \xi)$ to be the smallest number of open balls $B_{\epsilon e \widehat{C}(\omega)}(y) \subset \mathbb{R}^{d}$ centred at points $y \in K(\omega, \xi)$ that are needed to cover the compact set $K(\omega, \xi)$.

Lemma 3.6. a) The $N_{\epsilon}$ are $\mathcal{F} \times \mathcal{B}$-measurable.

b) For each $\omega \in \Omega$, the function $N_{\epsilon}(\omega,):. \Xi \rightarrow \mathbb{N}$ is upper semicontinuous. 
Fix $\epsilon \in(0, r], \omega \in \Omega_{k}$ and $\xi \in \Xi$, and denote $N=N_{\epsilon}(\omega, \xi)$. There are $y_{1}, \ldots, y_{N} \in K(\omega, \xi)$ such that $K(\omega, \xi) \subset \bigcup_{i=1}^{N} B_{\epsilon e} \widehat{C}(\omega)\left(y_{i}\right)$. As $h_{\omega, \xi}(K(\omega, \xi))=$ $K((\theta \ltimes g)(\omega, \xi))$, it follows from (3.5) that

$$
\begin{aligned}
K\left((\theta \ltimes g)^{k}(\omega, \xi)\right) & \subset \bigcup_{i=1}^{N} h_{\omega, \xi}^{k}\left(B_{\epsilon e} \widehat{C}(\omega)\right. \\
& \subset \bigcup_{i=1}^{N} B_{e^{-\eta} \epsilon e^{\widehat{C}\left(\theta^{k} \omega\right)}}\left(h_{\omega, \xi}^{k}\left(y_{i}\right)\right) \text { for } \mathbb{P} \text {-a.e. } \omega \in \Omega_{k}
\end{aligned}
$$

with points $h_{\omega, \xi}^{k}\left(y_{i}\right) \in K\left((\theta \ltimes g)^{k}(\omega, \xi)\right)$. Hence

$$
N_{\epsilon}\left((\theta \ltimes g)^{k}(\omega, \xi)\right) \leqslant N_{e^{-\eta} \epsilon}\left((\theta \ltimes g)^{k}(\omega, \xi)\right) \leqslant N_{\epsilon}(\omega, \xi) .
$$

Consider the restricted system $(\theta \ltimes g)^{k}{ }_{\mid \Omega_{k} \times \Theta}$ and denote the normalised probability measure $\mathbb{P}_{\mid \Omega_{k}}$ by $\mathbb{P}_{k}$. By Lemma 3.6, there is a subset $\Omega_{k}^{\prime} \subseteq \Omega_{k}$ of full measure such that the random sets $U_{\epsilon, \alpha}=\left\{(\omega, \xi) \in \Omega_{k} \times \Xi \mid N_{\epsilon}(\omega, \xi)<\alpha\right\}$ are open for all $\alpha \in \mathbb{R}$ and $\epsilon=e^{-p \eta} r$ with $p \in \mathbb{N}$. For measurability purposes we restrict to these countably many values of $\epsilon$ from now on. Let

$$
n_{\epsilon}(\omega)=\min \left\{\alpha \in \mathbb{N} \mid U_{\epsilon, \alpha}(\omega) \neq \emptyset\right\} .
$$

The measurability of $n_{\epsilon}$ follows easily from Lemma 4.1/d, Due to (3.6) we have $n_{\epsilon}\left(\theta^{k} \omega\right) \leqslant n_{\epsilon}(\omega)$ for $\mathbb{P}_{k}$-a.e. $\omega \in \Omega_{k}$, and thus the ergodicity of $\left(\theta^{k}, \mathbb{P}_{k}\right)$ implies that all $n_{\epsilon}$ are constant $\mathbb{P}_{k}$-a.e. We denote these constant integers by $n_{\epsilon}$ again. By the first inequality of (3.6), $n_{\epsilon} \leqslant n_{e^{-} \eta_{\epsilon}}$. But the second inequality of (3.6) implies that also $n_{e^{-\eta} \epsilon} \leqslant n_{\epsilon}$ for all $\epsilon \in(0, r]$, so that all $n_{\epsilon}$ coincide. Denote their common value by $n$.

Using (3.6) again, we see that the random open set $U_{r, n}$ is $(\theta \ltimes g)^{k}$-invariant and we have $U_{r, n}=U_{\epsilon, n}$ for all $\epsilon$. Similarly, for each integer $m>n$ the set $U_{r, m}$ is a non-empty $(\theta \ltimes g)^{k}$-invariant open random set and $U_{r, m}=U_{\epsilon, m}$ for all $\epsilon$. Since the random compact set $K \cap\left(\Omega_{k} \times M\right)$ is covered by these countably many random open sets $\left(U_{r, m}\right)_{m \in \mathbb{N}}$, we see that the following hold for $\mathbb{P}_{k}$-a.e. $\omega \in \Omega_{k}$ :

(1) $\# K(\omega, \xi)=n$ for all $\xi \in U_{r, n}$;

(2) $\sup \{\# K(\omega, \xi) \mid \xi \in \Xi\}<\infty$;

(3) $d\left(y, y^{\prime}\right) \geqslant c(\omega):=r e^{\widehat{C}(\omega)} / 2>0$ for all $\xi \in \Xi$ and any two different points $y, y^{\prime} \in K(\omega, \xi)$.

Let $A_{k}=U_{r, n}$. If $k=1$, then $A=A_{k}$ satisfies the assertions of the proposition. Otherwise, we let $A=\bigcup_{i=0}^{k-1}(\theta \ltimes g)^{i}\left(A_{k}\right)$. Then, as $\bigcup_{i=0}^{k-1} \theta^{k}\left(\Omega_{k}\right)=\Omega$ up to a set of $\mathbb{P}$-measure zero, as the $g_{\omega}^{i}$ are homeomorphisms, and as $h_{\omega, \xi}^{i}(K(\omega, \xi))=$ $K\left((\theta \ltimes g)^{i}(\omega, \xi)\right)$, assertions (1)-(3) carry over to $\mathbb{P}$-a.e. $\omega \in \Omega$.

Remark 3.7. If the second alternative " $K(\omega)=\emptyset$ for $\mathbb{P}$-a.e. $\omega$ " in the definition of minimality (Definition 1.3) is replaced by " $K(\omega)$ is nowhere dense for $\mathbb{P}$-a.e. $\omega$ ", $\theta \ltimes g$ is called transitive. Random transitivity seems to be a more subtle concept than random minimality; some of its aspects are discussed in Section 4 . Here we only note a version of Theorem 3.1 for random transitive homeomorphisms:

If the random homeomorphism in Theorem 3.1 is not minimal but only transitive, then there are a (non-random) integer $n>0$, a random $\theta \ltimes g$-invariant open dense 
set $A(\omega) \subseteq \Xi$ and a random variable $c(\omega)>0$ such that, for $\mathbb{P}$-a.e. $\omega \in \Omega$,

(i) $\# K(\omega, \xi)=n$ for all $\xi \in A(\omega)$,

(ii) the map $\xi \mapsto K(\omega, \xi)$ from $A(\omega)$ to $\mathcal{K}\left(\mathbb{R}^{d}\right)$ is continuous, and

(iii) for all $\xi \in \Xi$, any two different points $y, y^{\prime} \in K(\omega, \xi)$ have distance at least $c(\omega)$.

As in the proof of Theorem 3.1, the result is deduced from Proposition 3.4. The only difference is that the sets $A^{c}(\omega)$ are no longer empty, but nowhere dense, so that the open sets $A(\omega)$ are dense.

Proof of Lemma 3.5. For each $(\omega, \xi) \in \Omega \times \Xi$, the set $K(\omega, \xi)=\left\{y \in \mathbb{R}^{d}:(\xi, y) \in\right.$ $K(\omega)\}$ is compact because $K(\omega)$ is. Denote the Euclidean metric on $\mathbb{R}^{d}$ by dist and the metric on $\Xi$ by $\rho$. Define, for each $n>0$, a metric $d_{n}$ on $\Xi \times \mathbb{R}^{d}$ by $d_{n}\left((\xi, y),\left(\xi^{\prime}, y^{\prime}\right)\right)=\operatorname{dist}\left(y, y^{\prime}\right)+n \rho\left(\xi^{\prime}, \xi\right)$. Each $d_{n}$ generates the product topology on $\Xi \times \mathbb{R}^{d}$. By definition, $d_{n}((\xi, y), K(\omega)) \leqslant \operatorname{dist}(y, K(\omega, \xi))$ for all $n$. On the other hand, for each $n$ and $\omega$ there is $\left(\xi_{n}, y_{n}\right) \in \Xi \times \mathbb{R}^{d}$ such that $d_{n}((\xi, y), K(\omega))=$ $d_{n}\left((\xi, y),\left(\xi_{n}, y_{n}\right)\right)$. As $K(\omega)$ is compact, there is a subsequence $\left(\xi_{n_{i}}, y_{n_{i}}\right)$ converging to some $\left(\xi^{\prime}, y^{\prime}\right) \in K(\omega)$. If $\xi^{\prime} \neq \xi$, then obviously $\sup _{n} d_{n}((\xi, y), K(\omega))=$ $+\infty \geqslant \operatorname{dist}(y, K(\omega, \xi))$. If $\xi^{\prime}=\xi$, then $\sup _{n} d_{n}((\xi, y), K(\omega)) \geqslant \sup _{i} \operatorname{dist}\left(y, y_{n_{i}}\right) \geqslant$ $\operatorname{dist}\left(y, y^{\prime}\right) \geqslant \operatorname{dist}(y, K(\omega, \xi))$. Hence

$$
\sup _{n} d_{n}((\xi, y), K(\omega))=\operatorname{dist}(y, K(\omega, \xi)) .
$$

As $\omega \mapsto d_{n}((\xi, y), K(\omega))$ is measurable for all $(\xi, y) \in \Xi \times \mathbb{R}^{d}$ and $\xi \mapsto d_{n}((\xi, y), K(\omega))$ is continuous for all $\omega \in \Omega$ and $y \in \mathbb{R}^{d}$, it follows that $(\omega, \xi) \mapsto d_{n}((\xi, y), K(\omega))$ is measurable for all $y \in \mathbb{R}^{d}$; see [10, Lemma 1.5.2] or [14, Lemma 3.14]. Hence also dist $(y, K(\omega, \xi))$ is measurable so that $K(\omega, \xi)$ is indeed a random compact set. Finally, $h_{\omega, \xi}(K(\omega, \xi))=K((\theta \ltimes g)(\omega, \xi))$ follows from the observation that

$$
\begin{aligned}
y \in K(\omega, \xi) & \Leftrightarrow(\xi, y) \in K(\omega) \\
& \Leftrightarrow T_{\omega}(\xi, y)=\left(g_{\omega}(\xi), h_{\omega, \xi}(y)\right) \in K(\theta \omega) \\
& \Leftrightarrow h_{\omega, \xi}(y) \in K((\theta \ltimes g)(\omega, \xi))=K\left(\theta \omega, g_{\omega}(\xi)\right) .
\end{aligned}
$$

Proof of Lemma 3.6, a) By Lemma 3.5, $K$ is a random compact set over the base $(\Omega \times \Xi, \mathcal{F} \times \mathcal{B})$ so that there is a sequence $\left(a_{k}\right)_{k \in \mathbb{N}}$ of measurable maps $a_{k}: \Omega \times \Xi \rightarrow$ $\mathbb{R}^{d}$ such that $K(\omega, \xi)=\operatorname{closure}\left\{a_{k}(\omega, \xi) \mid k \in \mathbb{N}\right\}$ for all $(\omega, \xi) \in \Omega \times \Xi$ [14, Theorem III.30]; see also [10, Proposition 1.6.3].

Let $\epsilon>0$. For $n \in \mathbb{N}$ denote by $\mathcal{L}_{n}$ the family of subsets of $\mathbb{N}$ with $n$ elements. Then the sets

$$
V_{n}:=\bigcup_{L \in \mathcal{L}_{n}} \bigcap_{k \in \mathbb{N}} \bigcup_{\ell \in L}\left\{(\omega, \xi) \in \Omega \times \Xi \mid\left\|a_{k}(\omega, \xi)-a_{\ell}(\omega, \xi)\right\|<\epsilon\right\}
$$

are $\mathcal{F} \times \mathcal{B}$-measurable, and $N_{\epsilon}(\omega, \xi) \leqslant n$ if and only if $(\omega, \xi) \in V_{n}$. This proves the measurability of $N_{\epsilon}$.

b) Let $\omega \in \Omega$. In order to prove that the function $\xi \mapsto N_{\epsilon}(\omega, \xi)$ is upper semicontinuous, it suffices to observe that, for each $\alpha \in \mathbb{R}$, the set $\left\{\xi \in \Xi \mid N_{\epsilon}(\omega, \xi)<\alpha\right\}$ is open, because $K(\omega)$ is compact. 


\section{RANDOM MINIMALITY AND RANDOM TRANSITIVITY}

We start by collecting a few elementary, but useful facts.

Lemma 4.1. a) If $K$ is a random compact set, then $\operatorname{int}(K)$ (the fibrewise interior of $K$ ) is a random open set.

b) If $\theta \ltimes g$ is a random homeomorphism and $K$ is a random compact set, then $(\theta \ltimes g)(K)$ is a random compact set with fibres $g_{\theta^{-1} \omega}\left(K\left(\theta^{-1} \omega\right)\right)$.

c) If $K_{0}, K_{1}, \ldots$ are random compact sets, then $\bigcap_{n=0}^{\infty} K_{n}$ is a random compact set with fibres $\bigcap_{n=0}^{\infty} K_{n}(\omega)$.

d) If $A$ is a random open or closed set, then $\pi_{1}(A)=\{\omega \in \Omega \mid A(\omega) \neq \emptyset\}$ is $\mathcal{F}$-measurable.

Proof. 回) See [17, Chapter 3].

(b) There is a sequence $a_{0}, a_{1}, a_{2}, \ldots$ of measurable selections of $K$ such that $K(\omega)=\operatorname{closure}\left\{a_{k}(\omega): k \in \mathbb{N}\right\}$ for all $\omega \in \Omega$ [14, Theorem III.30]. Then the maps $g_{\theta^{-1}} .\left(a_{k}\left(\theta^{-1}.\right)\right)$ are measurable maps from $\Omega$ to $\Xi$ and $g_{\theta^{-1} \omega}\left(K\left(\theta^{-1} \omega\right)\right)=$ closure $\left\{g_{\theta^{-1} \omega}\left(a_{k}\left(\theta^{-1} \omega\right)\right) \mid k \in \mathbb{N}\right\}$, so that $(\theta \ltimes g)(K)$ is a random compact set by [14, Theorem III.30] again.

C) This is part of [14, Proposition III.4].

(d) For closed $A, \pi_{1}(A)$ is measurable by [10, Prop. 1.6.2]. If $A$ is open, then $\left(\pi_{1}(A)\right)^{c}=\left\{\omega \in \Omega \mid A^{c}(\omega)=\Xi\right\}=\bigcap_{G}\left\{\omega \in \Omega \mid A^{c}(\omega) \cap G \neq \emptyset\right\}$ where the intersection ranges over a countabale base $\{G\}$ of the topology of $\Xi$, and this set is measurable by [10, Prop. 1.6.2] again.

Definition 4.2. Let $\theta \ltimes g$ be a random homeomorphism on $\Omega \times \Xi$.

a) A random point $\xi$ is a measurable map $\xi: \Omega \rightarrow \Xi$.

b) The random point $\xi$ is a limit point of the sequence $\left(\xi_{n}\right)_{n \in \mathbb{N}}$ of random points if for each $\omega \in \Omega$ the point $\xi(\omega) \in \Xi$ is a limit point of the sequence $\left(\xi_{n}(\omega)\right)_{n \in \mathbb{N}}$.

c) The forward orbit of the random point $\xi$ is the sequence $\left(\xi_{n}\right)_{n \in \mathbb{N}}$ of random points defined by $\xi_{n}(\omega)=g_{\theta^{-n} \omega}^{n}\left(\xi\left(\theta^{-n} \omega\right)\right)$. The backward orbit is defined analogously by replacing $n$ with $-n$.

d) The omega limit set of a random point $\xi$ is the random compact set $\mho_{\xi}$ with fibres $\mho_{\xi}(\omega)=\bigcap_{k \in \mathbb{N}} \operatorname{closure}\left\{\xi_{n}(\omega) \mid n \geqslant k\right\}$ where $\left(\xi_{n}\right)_{n \in \mathbb{N}}$ is the orbit of $\xi$.

Remark 4.3. $\quad$ a) In view of [14, Theorem III.9] and Lemma 4.1, $\mho_{\xi}$ is indeed a random compact set.

b) $\mho_{\xi}$ is $(\theta \ltimes g)$-invariant.

c) A random point $\zeta$ is a limit point of the orbit of $\xi$ if and only if $\zeta$ is a measurable selection of $\mho_{\xi}$.

Recall the definition of a random minimal homeomorphism:

Definition 4.4. The random homeomorphism $\theta \ltimes g$ on $\Omega \times \Xi$ is minimal if each $(\theta \ltimes g)$-forward invariant random closed set $K$ obeys the following dichotomy:

either : $K(\omega)=\Xi$ for $\mathbb{P}$-a.e. $\omega$,

or $\quad: K(\omega)=\emptyset$ for $\mathbb{P}$-a.e. $\omega$. 
Lemma 4.5. Let $\theta \ltimes g$ be a random homeomorphism on $\Omega \times \Xi$. The following are equivalent:

(i) $\theta \ltimes g$ is minimal.

(ii) The dichotomy in Definition 4.4 holds for each $(\theta \ltimes g)$-backwards invariant random closed set $K$.

(iii) The dichotomy in Definition 4.4 holds for each $(\theta \ltimes g)$-invariant random closed set $K$.

(iv) For each random point $\xi$ holds: $\mho_{\xi}(\omega)=\Xi$ for $\mathbb{P}$-a.e. $\omega$.

Proof. (i) $\Rightarrow$ (iii) and (ii) $\Rightarrow$ (iii) are trivial.

(iii) $\Rightarrow$ (iv): For each random point $\xi, \mho_{\xi}$ is a non-empty $(\theta \ltimes g)$-invariant random compact set.

(iv) $\Rightarrow$ (i): Let $K$ be a $(\theta \ltimes g)$-forward invariant non-empty random closed set. It has at least one measurable selection $\xi$ [14, Theorem III.9]. Denote its orbit by $\left(\xi_{n}\right)_{n \in \mathbb{N}}$. Then all $\xi_{n}$ are measurable selections of $K$ as well since $\xi_{n}(\omega)=$ $g_{\theta^{-n} \omega}^{n}\left(\xi\left(\theta^{-n} \omega\right)\right) \in g_{\theta^{-n} \omega}^{n}\left(K\left(\theta^{-n} \omega\right)\right) \subseteq K(\omega)$. Hence, $\Xi=\mho_{\xi}(\omega) \subseteq K(\omega)$ for $\mathbb{P}$-a.e. $\omega$.

(iii) $\Rightarrow$ (ii): Let $K$ be a $(\theta \ltimes g)$-backward invariant random closed set. For each $\omega$ let $K^{\prime}(\omega)=\bigcap_{n=0}^{\infty}\left(g_{\omega}^{n}\right)^{-1}\left(K\left(\theta^{n} \omega\right)\right)$. As a decreasing intersection of non-empty random compact sets $K^{\prime}$ is a non-empty random compact set; see Lemma 4.1. It is invariant, because

$$
g_{\omega}\left(K^{\prime}(\omega)\right)=g_{\omega}\left(\bigcap_{n=1}^{\infty}\left(g_{\omega}^{n}\right)^{-1}\left(K\left(\theta^{n} \omega\right)\right)\right)=\bigcap_{n=0}^{\infty}\left(g_{\theta \omega}^{n}\right)^{-1}\left(K\left(\theta^{n} \theta \omega\right)\right)=K^{\prime}(\theta \omega) .
$$

Hence $K^{\prime}(\omega)=\Xi$ for $\mathbb{P}$-a.e. $\omega$. As $K^{\prime}(\omega) \subseteq K(\omega)$, the same holds for $K(\omega)$.

It is tempting to characterise random transitive sets in the same way. We will see, however, that the situation is more complicated.

Definition 4.6. The random homeomorphism $\theta \ltimes g$ on $\Omega \times \Xi$ is transitive if each $(\theta \ltimes g)$-forward invariant random closed set $K$ obeys the following dichotomy:

either : $K(\omega)=\Xi$ for $\mathbb{P}$-a.e. $\omega$,

or $\quad: K(\omega)$ is nowhere dense for $\mathbb{P}$-a.e. $\omega$.

In the same way as for Lemma 4.5, one proves:

Lemma 4.7. Let $\theta \ltimes g$ be a random homeomorphism on $\Omega \times \Xi$. The following are equivalent:

(i) $\theta \ltimes g$ is transitive.

(ii) The dichotomy in Definition 4.6 holds for each $(\theta \ltimes g)$-backwards invariant random closed set $K$.

(iii) The dichotomy in Definition 4.6 holds for each $(\theta \ltimes g)$-invariant random closed set $K$.

Recall that for a homeomorphism $g$ of a compact metric space $\Xi$, the following two statements are equivalent to topological transitivity: (1) Given any open sets $U, V \subseteq \Xi$ there exists $n \in \mathbb{N}$ with $g^{n}(U) \cap V \neq \emptyset$, and (2) there exists a point $x \in \Xi$ with dense orbit. 
The first of these equivalent characterisations can easily be carried over to random dynamical systems. Recall that we say a random set $R$ (open or compact) is non-empty if $\{\omega \in \Omega \mid R(\omega) \neq \emptyset\}$ has positive measure.

Lemma 4.8. A random homeomorphism $\theta \ltimes g$ on $\Omega \times \Xi$ is transitive if and only if

(T1) for all non-empty random open sets $U, V \subseteq \Omega \times \Xi$ there exists $n \in \mathbb{N}$ such that $(\theta \ltimes g)^{n}(U) \cap V$ is non-empty.

Proof. Suppose $\theta \ltimes g$ is transitive and $U, V \subseteq \Xi$ are non-empty random open sets. Assume for a contradiction that

$$
\mathbb{P}\left(\pi_{1}\left((\theta \ltimes g)^{n}(U) \cap V\right)\right)=0
$$

for all $n \in \mathbb{N}$. Let $\mathcal{U}=\bigcup_{n \in \mathbb{N}}(\theta \ltimes g)^{n}(U)$. Then $\mathcal{U}$ is forward invariant and the set

$$
\Omega^{\prime}=\{\omega \in \Omega \mid \mathcal{U}(\omega) \cap V(\omega)=\emptyset\}=\bigcup_{n \in \mathbb{N}} \pi_{1}\left((\theta \ltimes g)^{n}(U) \cap V\right)
$$

has measure zero. Hence, the backwards $(\theta \ltimes g)$-invariant random compact set $K=(\Omega \times \Xi) \backslash \mathcal{U}$ contains $V^{\prime}=V \backslash\left(\Omega^{\prime} \times \Xi\right)$, but is disjoint from $U$. By Lemma 4.7(ii), this contradicts the transitivity of $\theta \ltimes g$.

Conversely, suppose that (T1) holds and $K$ is a forward $(\theta \ltimes g)$-invariant compact set such that $\operatorname{int}(K(\omega)) \neq \emptyset \mathbb{P}$-a.s. Let $V=(\Omega \times \Xi) \backslash K$ and assume for a contradiction that $V$ is non-empty, such that we do not have $K(\omega)=\Xi$ for $\mathbb{P}$-a.e. $\omega \in \Omega$. Define the random open set $U$ by $U(\omega)=\operatorname{int}(K(\omega))$; see Lemma 4.1面, Then, by contradiction assumption, $U \subseteq K$ is non-empty, and due to the forward $(\theta \ltimes g)$-invariance of $K$ we have $(\theta \ltimes g)^{n}(U) \cap V \subseteq K \cap V=\emptyset$ for all $n \in \mathbb{N}$. This contradicts $(\mathrm{T} 1)$.

In order to generalise (2) to the random situation, one might hope to characterise random transitivity by the existence of a random point $\xi$ such that $\mho_{\xi}(\omega)=\Xi$ for $\mathbb{P}$-a.e. $\omega$. The following simple example shows that this cannot work: Let $\Omega=\Xi$ be a compact metric space and consider the random homeomorphism $\theta \ltimes g$ on $\Omega \times \Xi$ with a minimal homeomorphism $\theta: \Omega \rightarrow \Omega$, an ergodic probability $\mathbb{P}$ on $\Omega$ with full topological support, and $g_{\omega}(\xi)=\xi$ for all $(\omega, \xi)$. Except in the case of trivial $\Xi$, this is clearly not random transitive, because $(\Omega \times G)$ is an open $(\theta \ltimes g)$-invariant set for each open $G \subset \Xi$. On the other hand, $\xi(\omega)=\omega$ defines a random point with $\xi_{n}(\omega)=\xi\left(\theta^{-n} \omega\right)=\theta^{-n} \omega$ for all $\omega$ and $n$. Then, by minimality of $\theta$, we have $\mho_{\xi}(\omega)=\Xi$ for all $\omega \in \Omega$.

Hence, in order to make sense of (2) in a random setting we need a refined concept of 'dense orbit'. Given a random point $\xi$ and a set $A \subseteq \Omega$ of positive measure, we call the restriction of $\xi$ to $A$, denoted by $\xi^{A}: A \rightarrow \Xi, \omega \mapsto \xi(\theta)$, a subsection of $\xi$. We define $\xi_{n}^{A}: \theta^{n}(A) \rightarrow \Xi$ by $\xi_{n}^{A}(\omega)=g_{\theta^{-n} \omega}^{n}\left(\xi^{A}\left(\theta^{-n} \omega\right)\right)$ and define the omega limit $\mho_{\xi^{A}}$ of $\xi^{A}$ fibrewise as $\mho_{\xi^{A}}(\omega)=\bigcap_{k \in \mathbb{N}} \operatorname{closure}\left\{\xi_{n}^{A}(\omega) \mid n \geqslant k\right.$ s.t. $\left.\omega \in \theta^{n}(A)\right\}$.

Remark 4.9. The following observations are easy to prove.

a) $\mho_{\xi^{A}}$ is a random compact set.

b) $\mho_{\xi^{A}}$ is $(\theta \ltimes g)$-invariant.

c) Item (iv) in Lemma 4.5 can be replaced by (iv) $)^{\prime} \mho_{\xi^{A}}(\omega)=\Xi \mathbb{P}$-a.s. for every subsection $\xi^{A}$ of any random point $\xi$. 
Using this concept, we have the following.

Lemma 4.10. Suppose for the random homeomorphism $\theta \ltimes g$ there exists a random point $\xi$ such that $\mho_{\xi^{A}}(\omega)=\Xi \mathbb{P}$-a.s. for all subsections $\xi^{A}$ of $\xi$. Then $\theta \ltimes g$ is transitive.

Proof. Let $\xi$ be the random point with the above property. Suppose $K$ is a $(\theta \ltimes g)$ invariant random compact set such that $\{\omega \in \Omega \mid \operatorname{int}(K(\omega)) \neq \emptyset\}$ has positive measure. Let $U \subseteq K$ be the non-empty random open set defined by $U(\omega)=$ $\operatorname{int}(K(\omega))$.

As $\mho_{\xi}(\omega)=\Xi$ for $\mathbb{P}$-a.e. $\omega \in \Omega$, the set

$$
\left\{\omega \in \Omega \mid \exists n \in \mathbb{N}: \xi_{n}(\omega) \in U(\omega)\right\}=\bigcup_{n \in \mathbb{N}}\left\{\omega \in \Omega \mid \xi_{n}(\omega) \in U(\omega)\right\}
$$

has full measure. Hence, there exists $n \in \mathbb{N}$ such that $A^{\prime}=\left\{\omega \in \Omega \mid \xi_{n}(\omega) \in U(\omega)\right\}$ has positive measure. Then $A=\theta^{-n}\left(A^{\prime}\right)$ satisfies $\xi^{A}(\omega) \in K(\omega)$ for all $\omega \in A$, and consequently $\mho_{\xi^{A}} \subseteq K$. By assumption, this implies $K(\omega)=\Xi \mathbb{P}$-a.s. Since $K$ was an arbitrary $(\theta \ltimes g)$-invariant random compact set, Lemma 4.7(iii) implies that $\theta \ltimes g$ is transitive.

This suggests that a natural way to generalise (2) would be to show that random transitivity is equivalent to the existence of a random point $\xi$ whose subsections all have dense orbit (in the sense that $\mho_{\xi^{A}}=\Xi$ for $\mathbb{P}$-a.e. $\omega \in \Omega$ ). However, we are at the moment not able to prove this, nor to give a counterexample. A positive result may depend on additional assumptions on the base $(\Omega, \mathcal{B}, \mathbb{P}, \theta)$. We thus have to leave the following problem open.

Problem 4.11. Show that under suitable assumptions on $(\Omega, \mathcal{B}, \mathbb{P}, \theta)$ a transitive random homeomorphism $\theta \ltimes g$ has a random point $\xi$ whose subsections all have dense orbit.

Example 4.12. A natural class of examples of minimal random homeomorphisms is given by random circle rotations, although it needs a little work to give a rigorous proof of their minimality. We provide a sketch of the argument. Given a measurepreserving dynamical system $(\Omega, \mathcal{B}, \mathbb{P}, \theta)$ and a measurable function $\alpha: \Omega \rightarrow \mathbb{S}^{1}=$ $\{z \in \mathbb{C}|| z \mid=1\}$, we let $\Xi=\mathbb{S}^{1}$ and define a random homeomorphism $\theta \ltimes g^{\tau}$ with parameter $\tau \in \mathbb{S}^{1}$ by

$$
g_{\omega}^{\tau}(z)=\alpha(\omega) \tau z .
$$

It is easy to see that $\mathbb{P} \times \operatorname{Leb}_{\mathbb{S}^{1}}$ belongs to $\mathcal{M}_{\mathbb{P}}\left(\theta \ltimes g^{\tau}\right)$. Further, using the random Krylov-Bogolyubov Procedure [10, Theorem 1.5.8] it is possible to show that if $\mathbb{P} \times \operatorname{Leb}_{\mathbb{S}^{1}}$ is the unique measure in $\mathcal{M}_{\mathbb{P}}\left(\theta \ltimes g^{\tau}\right)$, then $\theta \ltimes g$ is minimal. An old result of Furstenberg [19, Lemma 2.1] states that the existence of further measures in $\mathcal{M}_{\mathbb{P}}\left(\theta \ltimes g^{\tau}\right)$ implies that the Koopman operator of $\theta$ has a unimodular measurable generalised eigenfunction $R$ in the sense that

$$
R(\theta \omega)=(\tau \alpha(\omega))^{n} R(\omega) \text { for some } n \in \mathbb{Z} .
$$

Note that while the result in [19] is stated for continuous and uniquely ergodic base transformations $\theta$, the proof also works in the general case. If, for a given $n \in \mathbb{Z}$, there are two numbers $\tau$ and $\tilde{\tau}$ for which this identity holds (with eigenfunctions $R$ and $\tilde{R}$ ), then $\tilde{R} R^{-1}$ is an eigenfunction of the Koopman operator with eigenvalue $\left(\tilde{\tau} \tau^{-1}\right)^{n}$. Now, if $\theta$ is weakly mixing, then the only $L^{2}$-eigenvalue of the associated 
Koopman operator is 1 , so $\tilde{\tau} \tau^{-1}$ is a root of unity. As a consequence, there exist only countably many $\tau \in \mathbb{S}^{1}$ such that $\mathcal{M}_{\mathbb{P}}\left(\theta \ltimes g^{\tau}\right)$ contains more than one measure. Hence, $g^{\tau}$ is minimal for all but countably many $\tau \in \mathbb{S}^{1}$.

Basically the same argument works when $\theta$ is a minimal rotation of a $d$-dimensional torus. In this case, it follows easily from Fourier analysis that any iterate of $\theta$ can only have countably many $L^{2}$-eigenvalues, such that again $g^{\tau}$ is minimal for all but countably many $\tau \in \mathbb{S}^{1}$.

\section{ACKNOWLEDGEMENTS}

The authors were supported by the German Research Council (the first author by Emmy-Noether-Project Ja 1721/2-1, and the second author by DFG-grant Ke 514/8-1). Further, this work is part of the activities of the Scientific Network "Skew product dynamics and multifractal analysis" (DFG-grant Oe 538/3-1).

\section{REFERENCES}

[1] N. F. Rulkov, M. M. Sushchik, L. S. Tsimring, and H. D. I. Abarbanel, Generalized synchronization of chaos in directionally coupled chaotic systems, Phys. Rev. E 51 (1995), 980-994.

[2] K. Pyragas, Weak and strong synchronization of chaos, Phys. Rev. E 54 (1996), R4508R4511.

[3] Arkady Pikovsky, Michael Rosenblum, and Jürgen Kurths, Synchronization, A universal concept in nonlinear sciences, Cambridge Nonlinear Science Series, vol. 12, Cambridge University Press, Cambridge, 2001. MR 1869044 (2002m:37001)

[4] G. Keller, H. Jafri, and R. Ramaswamy, Nature of weak generalized synchronization in chaotically driven maps, Physical Review E 87 (2013), 042913.

[5] A. J. Homburg, Synchronization in iterated function systems, preprint, 2013.

[6] Awadhesh Prasad, Surendra Singh Negi, and Ramakrishna Ramaswamy, Strange nonchaotic attractors, Internat. J. Bifur. Chaos Appl. Sci. Engrg. 11 (2001), no. 2, 291-309, DOI 10.1142/S0218127401002195. MR1830343 (2002b:37044)

[7] R. Sturman and J. Stark, Semi-uniform ergodic theorems and applications to forced systems, Nonlinearity 13 (2000), no. 1, 113-143, DOI 10.1088/0951-7715/13/1/306. MR1734626 (2000m:37041)

[8] V. Anagnostopoulou and T. Jäger, Nonautonomous saddle-node bifurcations: random and deterministic forcing, J. Differential Equations 253 (2012), no. 2, 379-399, DOI 10.1016/j.jde.2012.03.016. MR2921199

[9] Jaroslav Stark, Regularity of invariant graphs for forced systems, Ergodic Theory Dynam. Systems 19 (1999), no. 1, 155-199, DOI 10.1017/S0143385799126555. MR1677161 (99k:58140)

[10] Ludwig Arnold, Random dynamical systems, Springer Monographs in Mathematics, SpringerVerlag, Berlin, 1998. MR1723992 (2000m:37087)

[11] V. Anagnostopoulou, T. Jäger, and G. Keller, A model for the non-autonomous hopf bifurcation, Nonlinearity 28 (2015), 2587-2616.

[12] Sebastian J. Schreiber, On growth rates of subadditive functions for semiflows, J. Differential Equations 148 (1998), no. 2, 334-350, DOI 10.1006/jdeq.1998.3471. MR.1643183 (2000a:37004)

[13] Yongluo Cao, On growth rates of sub-additive functions for semi-flows: determined and random cases, J. Differential Equations 231 (2006), no. 1, 1-17, DOI 10.1016/j.jde.2006.08.016. MR2287874(2008k:37071)

[14] C. Castaing and M. Valadier, Convex analysis and measurable multifunctions, Lecture Notes in Mathematics, Vol. 580, Springer-Verlag, Berlin-New York, 1977. MR0467310 (57 \#7169)

[15] Jean-Pierre Aubin and Hélène Frankowska, Set-valued analysis, Systems \& Control: Foundations \& Applications, vol. 2, Birkhäuser Boston, Inc., Boston, MA, 1990. MR.1048347 (91d:49001)

[16] Yongluo Cao, Stefano Luzzatto, and Isabel Rios, Uniform hyperbolicity for random maps with positive Lyapunov exponents, Proc. Amer. Math. Soc. 136 (2008), no. 10, 3591-3600, DOI 10.1090/S0002-9939-08-09347-7. MR2415043 (2010h:37118) 
[17] Hans Crauel, Random probability measures on Polish spaces, Stochastics Monographs, vol. 11, Taylor \& Francis, London, 2002. MR,1993844 (2004e:60005)

[18] Gerhard Keller, Equilibrium states in ergodic theory, London Mathematical Society Student Texts, vol. 42, Cambridge University Press, Cambridge, 1998. MR1618769 (99e:28022)

[19] H. Furstenberg, Strict ergodicity and transformation of the torus, Amer. J. Math. 83 (1961), 573-601. MR0133429 (24 \#A3263)

Institut für Analysis, Technische Universität Dresden, Zellescher Weg 12-14, 01069 Dresden, Germany

Department Mathematik, Universität Erlangen-Nürnberg, 91058 Erlangen, Germany 